Pacific

Journal of

Mathematics

THE MODULI SPACE OF REAL ALGEBRAIC CURVES OF GENUS 2

Francisco-Javier CirRe

Volume 208 No. 1

January 2003 


\title{
THE MODULI SPACE OF REAL ALGEBRAIC CURVES OF GENUS 2
}

\author{
FRANCISCO-JAVIER CIRRE
}

\begin{abstract}
We give an explicit description of each of the five connected components of the moduli space of real algebraic curves of genus 2. This is done in terms of the coefficients of equations defining such curves. We also find the subsets consisting of curves with prescribed automorphism group.
\end{abstract}

\section{Introduction.}

Riemann's moduli problem could be formulated as that of describing the set of isomorphism classes of complex algebraic curves of a given topological type. Its real counterpart, i.e., the description of the set of real isomorphism classes of real algebraic curves, was initiated by Klein [8] and [9], who mainly worked with polynomial equations that define the real curve in question. The problem has acquired importance in the last three decades due to both the development of proper techniques of real algebraic geometry and the right definition of the "analytic" surface associated to a real algebraic curve. Such definition is given in [1] and the associated surface is known as Klein surface. This establishes the well-known coequivalence between real algebraic curves (algebraic objects) and Klein surfaces (geometric ones). A large number of works have appeared since; a good reference for many of them is [11].

Usual methods to deal with moduli spaces are based on Teichmüller ones. Different points of view can be considered, such as quasiconformal mappings, see for example $[\mathbf{1 2}, \mathbf{1 4}, \mathbf{7}]$, abelian varieties $[\mathbf{1 3}, \mathbf{1 5}]$ or non-euclidean crystallographic groups [10]. They rely upon the geometry of the surface associated to a curve. The approach here is more classical in the sense that we directly work with polynomial equations of curves.

By considering antianalytic involutions on Riemann surfaces of genus 0 and 1, Alling and Greenleaf classified all isomorphism classes of rational and elliptic real curves ([1], Thms. 1.9.4 and 1.9.8). So it is interesting to understand the situation in higher genera. In this paper we study the case of hyperelliptic real curves, which in particular covers the genus 2 case. Using coefficients of polynomial equations we describe the connected components of the space $\mathcal{M}_{2}^{\mathbb{R}}$ of isomorphism classes of real algebraic curves of genus 2 . 
We also find their subsets consisting of curves with prescribed automorphism group.

The paper is organized as follows. In Section 1 we recall some well-known facts about real curves. The reader may find them, e.g., in Chapters 3 and 5 in [14]. The second section deals with hyperelliptic curves. In it we develop a simple method to approach the problem of classifying hyperelliptic real algebraic curves of arbitrary genus up to birational isomorphism. The method is based on a detailed analysis of the action of real Möbius transformations on the sphere. It is worth pointing out how much information can be obtained from this simple method. It is applied in Sections 3 to 6 to the genus 2 case; namely, for each topological type $(2, k, \varepsilon)$ we find a bijection $\Psi_{(2, k, \varepsilon)}$ between a basic semialgebraic subset $\Delta_{(2, k, \varepsilon)}$ of $\mathbb{R}^{3}$ and the real moduli space $\mathcal{M}_{(2, k, \varepsilon)}$ of real algebraic curves of topological type $(2, k, \varepsilon)$. The bijection $\Psi_{(2, k, \varepsilon)}$ is given in terms of the branch points of the curve. The moduli space $\mathcal{M}_{(2, k, \varepsilon)}$ admits a semianalytic structure (see $[\mathbf{1 3}, \mathbf{6}]$ ) which is natural in the sense that the positions of the branch points could be chosen as analytic parameters for the curves. (In the complex case, the branch-point positions were the first (heuristic) method used by Riemann to predict the dimension $3 g-3$ of the moduli space of complex algebraic curves of genus $g$.) We exhibit this naturality in Section 7 by showing that each map $\Psi_{(2, k, \varepsilon)}$ is indeed real analytic.

\section{Preliminaries.}

A real algebraic curve of genus $g$ is a pair $(X, \sigma)$ where $X$ is a projective, smooth, irreducible complex algebraic curve of genus $g$ and $\sigma: X \rightarrow X$ is an antianalytic involution on $X$. Two real curves $(X, \sigma)$ and $(Y, \tau)$ are isomorphic if there exists an isomorphism $f: X \rightarrow Y$ such that $f \circ \sigma=\tau \circ f$. We denote by $\mathcal{M}_{g}^{\mathbb{R}}$ the moduli space of real algebraic curves of genus $g$ :

$$
\mathcal{M}_{g}^{\mathbb{R}}=\{\text { isomorphism classes of real algebraic curves of genus } g\} .
$$

Two isomorphic real curves are homeomorphic but the converse is not true. $((X, \sigma)$ and $(Y, \tau)$ are homeomorphic if $f \circ \sigma=\tau \circ f$ for some homeomorphism $f: X \rightarrow Y$.) Weichold [16] showed that the homeomorphism class of $(X, \sigma)$ is determined by its topological type $(g, k, \varepsilon)$, where $g$ is the genus of $X, k$ is the number of connected components of the real part $X_{\sigma}$ of $(X, \sigma)$, which is the fixed point set of $\sigma$, and $\varepsilon=1$ if $X-X_{\sigma}$ is connected and 0 otherwise. For each value of $g$, there are exactly $[(3 g+4) / 2]$ topologically different real algebraic curves of genus $g$, where $[r]$ stands for the integer part of $r$. For each admissible triple $(g, k, \varepsilon)$ we write

$$
\mathcal{M}_{(g, k, \varepsilon)}=\left\{(X, \sigma) \in \mathcal{M}_{g}^{\mathbb{R}}:(X, \sigma) \text { has topological type }(g, k, \varepsilon)\right\},
$$


where we have used $(X, \sigma)$ to denote both a real algebraic curve and its isomorphism class. This convention will be assumed throughout this paper. For abbreviation, a real algebraic curve of topological type $(g, k, \varepsilon)$ will be called a $(g, k, \varepsilon)$-curve. The usual topology on $\mathcal{M}_{g}^{\mathbb{R}}$ makes the sets $\mathcal{M}_{(g, k, \varepsilon)}$ be the connected components of $\mathcal{M}_{g}^{\mathbb{R}}$. For example, $\mathcal{M}_{2}^{\mathbb{R}}$ has the following 5 connected components:

$$
\mathcal{M}_{2}^{\mathbb{R}}=\mathcal{M}_{(2,3,0)} \cup \mathcal{M}_{(2,2,1)} \cup \mathcal{M}_{(2,1,1)} \cup \mathcal{M}_{(2,1,0)} \cup \mathcal{M}_{(2,0,1)} .
$$

In terms of Klein surfaces $(2,3,0)$-curves correspond to spheres with 3 holes, $(2,2,1)$-curves to projective planes with 2 holes, $(2,1,1)$-curves to connected sums of two projective planes with a hole, $(2,1,0)$-curves to tori with a hole and $(2,0,1)$ to connected sums of three projective planes.

A natural partition of the moduli space $\mathcal{M}_{(g, k, \varepsilon)}$ arises when considering its subsets $\mathcal{M}_{(g, k, \varepsilon)}(H)$ formed by curves with the same automorphism group $H$ :

$$
\mathcal{M}_{(g, k, \varepsilon)}(H)=\left\{(X, \sigma) \in \mathcal{M}_{(g, k, \varepsilon)}: \operatorname{Aut}(X, \sigma)=H\right\},
$$

where $\operatorname{Aut}(X, \sigma)=\{f: X \rightarrow X: f$ analytic selfhomeomorphism such that $f \circ \sigma=\sigma \circ f\}$ is the automorphism group of $(X, \sigma)$. By means of combinatorial methods, the list of groups which are realized as the automorphism groups of real algebraic curves of genus 2 was calculated in [2] for $(2,0,1)$ curves and in $[\mathbf{3}]$ for the rest of cases. As a by-product we obtain here the same results although in a very different way. We will denote the cyclic group of order $N$ by $C_{N}$ and the dihedral group of order $2 N$ by $D_{N}$. Thus $D_{2}$ stands for the non-cyclic group of order 4 .

\section{Hyperelliptic real algebraic curves.}

A real algebraic curve $(X, \sigma)$ of genus $g \geq 2$ is hyperelliptic if so is the complex curve $X$, that is, if $X$ admits a meromorphic function of degree 2 . Throughout this paper we represent $X$ by its affine plane model

$$
X=\left\{(x, y) \in \mathbb{C}^{2}: y^{2}=P_{X}(x):=\left(x-e_{1}\right) \ldots\left(x-e_{2 g+1+\delta}\right)\right\}
$$

with $e_{i} \neq e_{j}$ and $\delta=0$ or 1 . In this model we identify the characteristic elements of a hyperelliptic curve. First, a meromorphic function of degree 2 is given by the projection $\pi_{X}:(x, y) \mapsto x$ onto the Riemann sphere $\widehat{\mathbb{C}}:=\mathbb{C} \cup\{\infty\}$. Its branch points are thus the roots of $P_{X}$ and possibly $\infty$. They constitute what we call (by abuse of language) the branch point set of $X$,

$$
B_{X}= \begin{cases}\left\{e_{1}, \ldots, e_{2 g+2}\right\} & \text { if } \delta=1 \\ \left\{e_{1}, \ldots, e_{2 g+1}, \infty\right\} & \text { if } \delta=0\end{cases}
$$

The automorphism interchanging the two sheets of $\pi_{X}$ is known as the hyperelliptic involution $h_{X}:(x, y) \mapsto(x,-y)$. It is central in $\operatorname{Aut}(X, \sigma)$. 
Let $Y=\left\{w^{2}=P_{Y}(z)\right\}$ be another hyperelliptic curve and $B_{Y}$ its branch point set. Every birational isomorphism $f: X \rightarrow Y$ induces a Möbius transformation $\hat{f}: \widehat{\mathbb{C}} \rightarrow \widehat{\mathbb{C}}$ which maps $B_{X}$ onto $B_{Y}$. In fact, $\hat{f}$ is defined by $\hat{f}: \pi_{X}(p) \mapsto \pi_{Y}(f(p))$ for any $p \in X$.

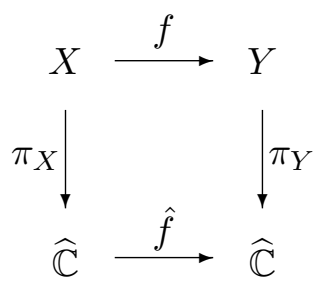

Conversely, every Möbius transformation $m: \widehat{\mathbb{C}} \rightarrow \widehat{\mathbb{C}}$ which maps $B_{X}$ onto $B_{Y}$ induces exactly two birational isomorphisms $f_{1}, f_{2}: X \rightarrow Y$ such that $\hat{f}_{i}=m, i=1,2$ (in fact, $f_{2}=f_{1} \circ h_{X}=h_{Y} \circ f_{1}$ ). We call these isomorphisms liftings of $m$. Their formulae can be calculated explicitly, [4].

Liftings of Möbius transformations. Writing

$$
m(x)=\frac{a x+b}{c x+d} \text { with } \operatorname{det} m:=a d-b c \neq 0,
$$

we have:

- If $\infty \in B_{X}$ and $m(\infty)=\infty$ then

$$
f_{1}(x, y)=\left(\frac{a x+b}{d}, y \cdot\left(\frac{a}{d}\right)^{g} \frac{\sqrt{\operatorname{det} m}}{d}\right) .
$$

- If $\infty \in B_{X}$ and $m(\infty) \neq \infty$ then

$$
f_{1}(x, y)=\left(\frac{a x+b}{c x+d}, \frac{y \cdot c^{g}}{(c x+d)^{g+1}} \sqrt{-\operatorname{det} m \cdot P_{Y}^{\prime}(m(\infty))}\right),
$$

where $P_{Y}^{\prime}$ denotes the derivative of $P_{Y}$.

- If $\infty \notin B_{X}$ and $m(\infty)=\infty$ then

$$
f_{1}(x, y)=\left(\frac{a x+b}{d}, y \cdot\left(\frac{a}{d}\right)^{g+1}\right) .
$$

- If $\infty \notin B_{X}$ and $m(\infty) \neq \infty$ then

$$
f_{1}(x, y)=\left(\frac{a x+b}{c x+d}, \frac{y \cdot c^{g+1}}{(c x+d)^{g+1}} \sqrt{P_{Y}(m(\infty))}\right) .
$$

We now return to real algebraic curves. If the hyperelliptic complex curve $Y$ admits an antianalytic involution $\tau$ then its branch point set $B_{Y}$ is preserved by the antianalytic involution $\hat{\tau}: \widehat{\mathbb{C}} \rightarrow \widehat{\mathbb{C}}$ induced by $\tau$. Further, $\hat{\tau}$ is conjugate to either the complex conjugation $x \mapsto \bar{x}$ or the antipodal map $x \mapsto-1 / \bar{x}([\mathbf{1}]$, Thm 1.9.4). In the latter case $Y$ has odd genus (see 2.2.(a) below). Since we are interested here in genus 2 curves, we only have to 
consider those $(Y, \tau)$ such that $\hat{\tau}$ is conjugate to the complex conjugation. The next lemma provides suitable plane models for such curves, [4].

Lemma 2.1. With the above notations, each hyperelliptic real curve $(Y, \tau)$ with $\hat{\tau}(x)=\bar{x}$ is isomorphic to one and only one of the following:

(i) $\left(X=\left\{y^{2}=P_{X}(x)\right\}, \sigma_{X}\right)$ where $P_{X}$ is a monic real polynomial without multiple roots, or

(ii) $\left(X=\left\{y^{2}=-P_{X}(x)\right\}, \sigma_{X}\right)$ where $P_{X}$ is a monic real polynomial without multiple roots and without real roots,

and in both cases, $\sigma_{X}:(x, y) \mapsto(\bar{x}, \bar{y})$.

Note that curves of type (ii) have empty real part $X_{\sigma}$ since $P_{X}$ is always positive on $\mathbb{R}$. On the contrary, curves of type (i) have nonempty real part. The complete topological description of any hyperelliptic real curve is given in the next lemma. It may be seen as a reformulation of results in [5], Section 6 adapted to our situation.

\section{Topological Classification Lemma 2.2.}

(a) Let $\left(X=\left\{y^{2}=P_{X}\right\}, \tau\right)$ be a hyperelliptic real curve such that the antianalytic involution that $\tau$ induces in $\widehat{\mathbb{C}}$ is conjugate to $\hat{\tau}: x \mapsto$ $-1 / \bar{x}$. Then its topological type is $(g, 0,1)$ with $g=\left[\left(\operatorname{deg} P_{X}-1\right) / 2\right]$ odd.

(b) Let $\left(X=\left\{y^{2}=P_{X}(x)\right\}, \sigma_{X}\right)$ be a hyperelliptic real curve of type (i) in the above lemma and let $z_{\mathbb{R}}$ be the number of real roots of $P_{X}$. Then its topological type is $(g, k, \varepsilon)$ with $g=\left[\left(\operatorname{deg} P_{X}-1\right) / 2\right]$ and

(b.1) $k=g+1, \varepsilon=0$ if $z_{\mathbb{R}}=\operatorname{deg} P_{X}$,

(b.2) $k=\left[\left(z_{\mathbb{R}}+1\right) / 2\right]$ and $\varepsilon=1$ if $0<z_{\mathbb{R}}<\operatorname{deg} P_{X}$,

(b.3) $k=1, \varepsilon=0$ if $z_{\mathbb{R}}=0$ and $g$ is even,

(b.4) $k=2, \varepsilon=0$ if $z_{\mathbb{R}}=0$ and $g$ is odd.

(c) Let $\left(X=\left\{y^{2}=-P_{X}(x)\right\}, \sigma_{X}\right)$ be a hyperelliptic real curve of type (ii) in the above lemma. Then its topological type is $(g, 0,1)$ with $g=$ $\left(\operatorname{deg} P_{X}-2\right) / 2$.

Since for curves of type (i) or (ii) (the only ones to be considered throughout this paper), the antianalytic involution is the complex conjugation, we will omit it and in the sequel we will simply say that $X$ is a real curve.

Let us return to the problem of classifying hyperelliptic curves up to isomorphism. Recall that a necessary and sufficient condition for two complex hyperelliptic curves to be isomorphic is the existence of a (complex) Möbius transformation mapping the branch point set of one of them onto that of the other. However, the situation in the real case is more complicated: The existence of a real Möbius transformation mapping $B_{X}$ onto $B_{Y}$ does not assure $X$ and $Y$ to be isomorphic. This obstruction in the real case is detected by means of the formulae of liftings (see above). A case by case examination 
of such formulae proves the following lemma. (A similar result for curves appearing in (a) of the Topological Classification Lemma is given in [4].)

$\mathbb{R}$-lifting Lemma 2.3. Let $X$ and $Y=\left\{y^{2}=P_{Y}(x)\right\}$ be two hyperelliptic real curves of type (i) in Lemma 2.1 and let $m$ be a real Möbius transformation mapping the branch point set of $X$ onto that of $Y$. The liftings of $m$ are isomorphisms between $X$ and $Y$ if and only if:

- $\operatorname{det} m>0$ if $\infty \in B_{X}$ and $m(\infty)=\infty$;

- $\operatorname{det} m \cdot P_{Y}^{\prime}(m(\infty))<0$ if $\infty \in B_{X}$ and $m(\infty) \neq \infty$;

- always if $\infty \notin B_{X}$ and $m(\infty)=\infty$;

- $P_{Y}(m(\infty))>0$ if $\infty \notin B_{X}$ and $m(\infty) \neq \infty$.

The same holds true if both $X$ and $Y$ are of type (ii).

In either case we will say that $m$ has real liftings.

Isomorphisms between $X$ and $Y$ become automorphisms of $X$ when $Y=$ $X$. The automorphism group Aut $X$ of $X$ consists of the liftings of those real Möbius transformations preserving $B_{X}$ which, in addition, have real liftings. We denote by Aut $X_{\widehat{\mathbb{C}}}$ the group of such Möbius transformations:

Aut $X_{\widehat{\mathbb{C}}}:=\left\{m: m\right.$ real, $m\left(B_{X}\right)=B_{X}$ and $m$ has real liftings $\}$.

Results of this section are applied to the particular case of genus 2 curves. In each of the following sections we give an explicit description of each $\mathcal{M}_{(2, k, \varepsilon)}$. We develop in detail only the case of $(2,3,0)$-curves. In the rest of the cases, proofs are outlined.

\section{Moduli of $(2,3,0)$-curves.}

The Topological Classification Lemma shows that all the branch points of a $(2,3,0)$-curve lie on the real line $\mathbb{R} \cup\{\infty\}$. An easy consequence of the $\mathbb{R}$-lifting lemma is that $\infty$ may be fixed as one of them. Moreover, we have the following:

Proposition 3.1. Each $(2,3,0)$-curve is isomorphic to another of the form

$$
X(a, b, c)=\left\{y^{2}=P_{X(a, b, c)}(x):=x(x-1)(x-a)(x-b)(x-c)\right\}
$$

with $0<a<b<c<1$.

Proof. Let $B_{Y}=\left\{e_{1}, \ldots, e_{5}, \infty\right\}$ with $e_{1}<\cdots<e_{5}$ be the branch point set of a $(2,3,0)$-curve $Y$. The Möbius transformation $m: x \mapsto\left(x-e_{1}\right) /\left(e_{5}-e_{1}\right)$ maps $B_{Y}$ onto $B_{X}=\left\{0, a:=m\left(e_{2}\right), b:=m\left(e_{3}\right), c:=m\left(e_{4}\right), 1, \infty\right\}$ with $0<a<b<c<1$. So the curve $X(a, b, c)$ we are looking for is the curve which ramifies over $B_{X}$ and given by the above equation. Indeed, it follows from the $\mathbb{R}$-lifting lemma that a lifting of $m$ makes $X$ and $Y$ isomorphic. 
Let $T$ be the following open subset of $\mathbb{R}^{3}$ :

$$
T=\left\{(a, b, c) \in \mathbb{R}^{3}: 0<a<b<c<1\right\} .
$$

The above proposition may be restated by saying that the mapping from $T$ to $\mathcal{M}_{(2,3,0)}$ given by

$$
\begin{array}{ccc}
T & \rightarrow \mathcal{M}_{(2,3,0)} \\
(a, b, c) & \mapsto X(a, b, c)
\end{array}
$$

is surjective. Thus, in order to describe $\mathcal{M}_{(2,3,0)}$ we have to find the fibres of this mapping. It turns out that they are orbits of points under the action of a finite group acting on $T$.

Proposition 3.2. Let $G$ be the dihedral group of order 6 generated by the involutions

$$
\alpha:(a, b, c) \mapsto\left(a, \frac{a}{c}, \frac{a}{b}\right) \quad \text { and } \beta:(a, b, c) \mapsto\left(\frac{b-c}{b-1}, \frac{a-c}{a-1}, c\right) .
$$

Then, with the above notations, $X\left(a^{\prime}, b^{\prime}, c^{\prime}\right)$ is isomorphic to $X(a, b, c)$ if and only if $\left(a^{\prime}, b^{\prime}, c^{\prime}\right)=\gamma(a, b, c)$ for some $\gamma \in G$.

Proof. We first have to determine all the real Möbius transformations which map $B_{X(a, b, c)}=\{\infty, 0, a, b, c, 1\}$ onto $B_{X\left(a^{\prime}, b^{\prime}, c^{\prime}\right)}=\left\{\infty, 0, a^{\prime}, b^{\prime}, c^{\prime}, 1\right\}$. If $m$ is such a transformation then its restriction to $\mathbb{R}-\left\{m^{-1}(\infty)\right\}$ is either strictly increasing or strictly decreasing according to the sign of its determinant. Therefore, the images by $m$ of the branch points of $X(a, b, c)$ are completely determined by the image of one of them and by the increasing or decreasing nature of $\mathrm{m}$. This gives 12 Möbius transformations, one for each choice of, e.g., $m(\infty)$ and the sign of det $m$. We then have to apply the $\mathbb{R}$-lifting lemma in order to find those having real liftings. For that we compute the sign of $P_{X\left(a^{\prime}, b^{\prime}, c^{\prime}\right)}^{\prime}$ at $m(\infty)$ (if $m(\infty) \neq \infty$ ). It appears in the fourth column of Table 1. In the fifth one we write "yes" or "no" according to whether $m$ has real liftings or not.

We next calculate the formula of each Möbius transformation having real liftings. In order to express it in terms of $a, b$ and $c$ we use the data $m_{j}^{-1}(0), m_{j}^{-1}(\infty)$ and $m_{j}^{-1}(1)$. We obtain the following six formulae:

$$
\begin{aligned}
& m_{1}(x)=x ; \quad m_{4}(x)=\frac{a}{x} ; \quad m_{5}(x)=\frac{b-c}{b-1} \cdot \frac{x-1}{x-c} ; \\
& m_{8}(x)=\frac{c-b}{c-a} \cdot \frac{x-a}{x-b} ; \quad m_{9}(x)=\frac{a}{b} \cdot \frac{x-b}{x-a} ; \quad m_{12}(x)=\frac{x-c}{x-1} .
\end{aligned}
$$

Calculating finally $m_{j}\left(B_{X(a, b, c)}\right)$ for each $m_{j}$ and arranging in increasing order the images different to 0,1 and $\infty$, we get the points $\left(a^{\prime}, b^{\prime}, c^{\prime}\right)$ with $0<$ $a^{\prime}<b^{\prime}<c^{\prime}<1$ we are looking for. They are precisely the images of $(a, b, c)$ under the elements of the group $G$ in the statement of the proposition. 


\section{Table 1}

\begin{tabular}{|c|c|c|c|c|}
\hline & $m_{j}(\infty)$ & $\operatorname{det} m_{j}$ & $P_{X\left(a^{\prime}, b^{\prime}, c^{\prime}\right)}^{\prime}\left(m_{j}(\infty)\right)$ & real liftings \\
\hline$m_{1}$ & $\infty$ & + & & Yes \\
\hline$m_{2}$ & $\infty$ & - & & No \\
\hline$m_{3}$ & 0 & + & + & No \\
\hline$m_{4}$ & 0 & - & + & Yes \\
\hline$m_{5}$ & $a^{\prime}$ & + & - & Yes \\
\hline$m_{6}$ & $a^{\prime}$ & - & - & No \\
\hline$m_{7}$ & $b^{\prime}$ & + & + & No \\
\hline$m_{8}$ & $b^{\prime}$ & - & + & Yes \\
\hline$m_{9}$ & $c^{\prime}$ & + & - & Yes \\
\hline$m_{10}$ & $c^{\prime}$ & - & - & No \\
\hline$m_{11}$ & 1 & + & + & No \\
\hline$m_{12}$ & 1 & - & + & Yes \\
\hline
\end{tabular}

As a consequence, each isomorphism class of a $(2,3,0)$-curve is represented by one and only one point of the quotient space $T / G$. The next proposition describes a fundamental set for the action of $G$ on $T$.

Proposition 3.3. The mapping $(a, b, c) \mapsto \operatorname{orbit}(a, b, c)$ is a bijection between the subset $\Delta$ of $T$ given by $\Delta=\left\{b c \leq a \leq \frac{b-c}{b-1}\right\}$ and the quotient $T / G$.

Proof. The generating involutions $\alpha$ and $\beta$ act as two reflections with respect to two surfaces of $T$ intersecting along a curve of $T$. More precisely, the fixed point set of $\alpha$ is the surface of $T \operatorname{fix}(\alpha)=\{a=b c\}$, and the two semispaces in which it divides $T$ are interchanged by $\alpha$, i.e., $(a, b, c) \in\{a>b c\}$ if and only if $\alpha(a, b, c) \in\{a<b c\}$. Similarly, $(a, b, c) \in\{a>(b-c) /(b-1)\}$ if and only if $\beta(a, b, c) \in\{a<(b-c) /(b-1)\}$, where $\{a=(b-c) /(b-1)\}$ is the fixed point set of $\beta$. Straightforward calculations show that the intersection $\{a \geq b c\} \cap\{a \leq(b-c) /(b-1)\}$ is a fundamental set $\Delta$ for the action of $G$ on $T$.

Summarizing, we have the following:

Theorem 3.4. The mapping

$$
\Psi:(a, b, c) \mapsto X(a, b, c)=\left\{y^{2}=x(x-1)(x-a)(x-b)(x-c)\right\}
$$

is a bijection between

$$
\Delta=\left\{(a, b, c) \in \mathbb{R}^{3}: 0<a<b<c<1, \quad b c \leq a \leq \frac{b-c}{b-1}\right\} \quad \text { and } \mathcal{M}_{(2,3,0)} .
$$


The next step for a better description of $\mathcal{M}_{(2,3,0)}$ is to identify in $\Delta$ its strata, i.e., its subsets $\mathcal{M}_{(2,3,0)}(H)$ consisting of curves whose automorphism group is $H$. It is clear that points in $T$ with nontrivial $G$-stabilizer represent curves with "nontrivial" automorphism group, i.e., other than $\left\langle h_{X}\right\rangle$. Hence, the strata of $\mathcal{M}_{(2,3,0)}$ are described in terms of fixed point sets of elements of $G$. In addition, we can calculate explicitly the automorphism group of each $(2,3,0)$-curve since we know all the Möbius transformations which permute its branch points.

Proposition 3.5. The subsets $D=\left\{b c<a<\frac{b-c}{b-1}\right\}, S=\left\{b c=a<\frac{b-c}{b-1}\right\} \cup$ $\left\{b c<a=\frac{b-c}{b-1}\right\}$ and $C=\left\{b c=a=\frac{b-c}{b-1}\right\}$ correspond, via the bijection $\Psi$ decribed above, to $\mathcal{M}_{(2,3,0)}\left(C_{2}\right), \mathcal{M}_{(2,3,0)}\left(D_{2}\right)$ and $\mathcal{M}_{(2,3,0)}\left(D_{6}\right)$ respectively. These are the only strata of $\mathcal{M}_{(2,3,0)}$. Furthermore, the explicit formulae of the automorphisms of the curves belonging to each stratum are given.

Proof. Let $m$ be any of the 6 Möbius transformations with real liftings we find in Proposition 3.2. We just have to calculate which conditions $a, b$ and $c$ must fulfill so that $m\left(B_{X(a, b, c)}\right)$ coincides with $B_{X(a, b, c)}$. With the same notations:

- $m_{4}(x)=\frac{a}{x}$ preserves $B_{X(a, b, c)}$ if and only if $a=b c$.

- $m_{5}(x)=\frac{b-c}{b-1} \cdot \frac{x-1}{x-c}$ preserves $B_{X(a, b, c)}$ if and only if $b c=a=\frac{b-c}{b-1}$.

- $m_{8}(x)=\frac{c-b}{c-a} \cdot \frac{x-a}{x-b}$ preserves $B_{X(a, b, c)}$ if and only if $a=1+c-\frac{c}{b}$. This surface intersects $\Delta$ in the curve $b c=a=\frac{b-c}{b-1}$; therefore $m_{8}$ preserves $B_{X(a, b, c)}$ if and only if $b c=a=\frac{b-c}{b-1}$.

- $m_{9}(x)=\frac{a}{b} \cdot \frac{x-b}{x-a}$ preserves $B_{X(a, b, c)}$ if and only if $b c=a=\frac{b-c}{b-1}$.

- $m_{12}(x)=\frac{x-c}{x-1}$ preserves $B_{X(a, b, c)}$ if and only if $a=\frac{b-c}{b-1}$.

Recall that the group of Möbius transformations with real liftings that preserve $B_{X(a, b, c)}$ is denoted by $\operatorname{Aut} X(p)_{\widehat{\mathbb{C}}}$, where $p=(a, b, c)$ for short. Let

$$
f:(x, y) \mapsto\left(\frac{a}{x}, \frac{-y a^{3 / 2}}{x^{3}}\right) \text { and } g:(x, y) \mapsto\left(\frac{x-c}{x-1}, \frac{y(1-c)^{3 / 2}}{(x-1)^{3}}\right)
$$

be liftings of $m_{4}$ and $m_{12}$, respectively. As a consequence of the above computations:

(i) If $p \in D=\left\{b c<a<\frac{b-c}{b-1}\right\}$ then none of the above Möbius transformations preserves $B_{X(p)}$, i.e., $\operatorname{Aut} X(p)_{\widehat{\mathbb{C}}}=\left\{\operatorname{id}_{\widehat{\mathbb{C}}}\right\}$ and so $\operatorname{Aut} X(p)=$ $\left\langle h_{X}\right\rangle \simeq C_{2}$.

(ii) If $p \in\left\{b c=a<\frac{b-c}{b-1}\right\}$ then $\operatorname{Aut} X(p)_{\widehat{\mathbb{C}}}=\left\{\operatorname{id}_{\widehat{\mathbb{C}}}, m_{4}\right\}$ and so $\operatorname{Aut} X(p)=$ $\left\langle h_{X}, f\right\rangle \simeq D_{2}$. If $p \in\left\{b c<a=\frac{b-c}{b-1}\right\}$ then $\operatorname{Aut} X(p)_{\widehat{\mathbb{C}}}=\left\{\mathrm{id}_{\widehat{\mathbb{C}}}, m_{12}\right\}$ and so $\operatorname{Aut} X(p)=\left\langle h_{X}, g\right\rangle \simeq D_{2}$.

(iii) If $p \in C=\left\{b c=a=\frac{b-c}{b-1}\right\}$ then $\operatorname{Aut} X(p)_{\widehat{\mathbb{C}}}=\left\{\mathrm{id}_{\widehat{\mathbb{C}}}, m_{4}, m_{5}, m_{8}, m_{9}\right.$, $\left.m_{12}\right\}$. It turns out that $\operatorname{Aut} X(p)=\langle f, g\rangle \simeq D_{6}$. 
Figure 1 illustrates what $\Delta$ looks like (dashed lines do not belong to $\Delta$ ). In it we identify its subsets corresponding to the strata of $\mathcal{M}_{(2,3,0)}$. The stratum $\mathcal{M}_{(2,3,0)}\left(C_{2}\right)$ corresponds to the interior of $\Delta$ in $T$, which is the domain bounded by the two shadowed surfaces.

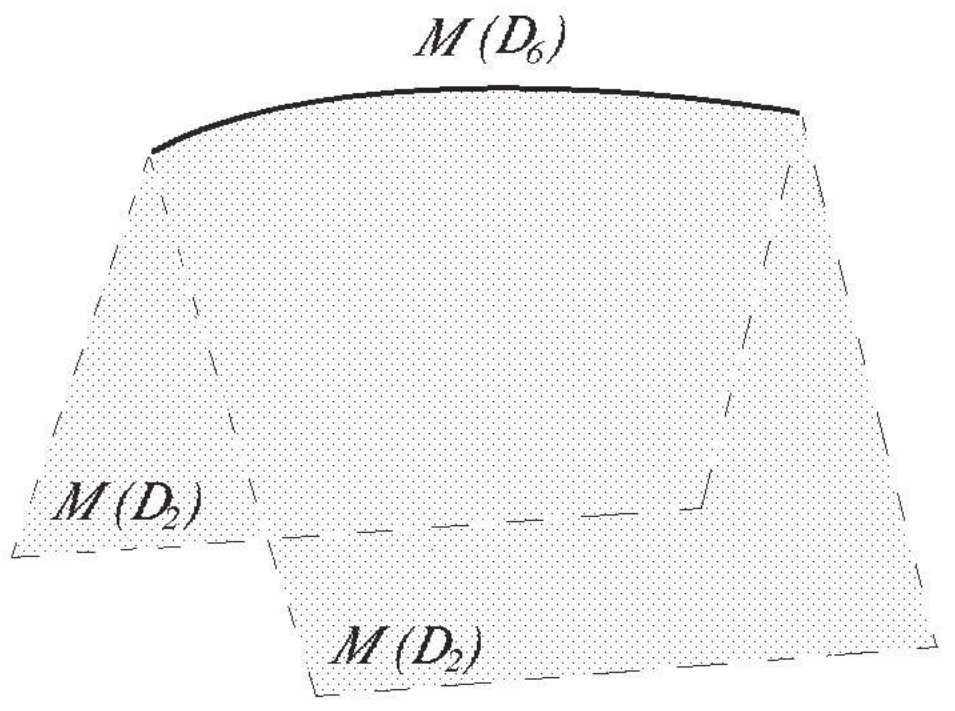

Figure 1. The moduli space of $(2,3,0)$-curves.

\section{Moduli of $(2,2,1)$-curves.}

By the Topological Classification Lemma, exactly 2 of the 6 branch points of a $(2,2,1)$-curve are complex non-real. It is easy to see that we may fix $i:=\sqrt{-1},-i$ and $\infty$ as branch points.

Proposition 4.1. Write $T=\left\{(a, b, c) \in \mathbb{R}^{3}: a<b<c\right\}$. The mapping $T \rightarrow \mathcal{M}_{(2,2,1)}$ given by $(a, b, c) \mapsto X(a, b, c)=\left\{y^{2}=\left(x^{2}+1\right)(x-a)(x-\right.$ $b)(x-c)\}$ is surjective.

Its fibres are orbits under the action of a finite group acting on $T$.

Proposition 4.2. Let $G$ be the dihedral group of order 4 generated by the involutions

$\alpha:(a, b, c) \mapsto\left(a, \frac{a c+1}{c-a}, \frac{a b+1}{b-a}\right)$ and $\beta:(a, b, c) \mapsto\left(\frac{b c+1}{b-c}, \frac{a c+1}{a-c}, c\right)$.

Then, with the above notations, $X\left(a^{\prime}, b^{\prime}, c^{\prime}\right)$ is isomorphic to $X(a, b, c)$ if and only if $\left(a^{\prime}, b^{\prime}, c^{\prime}\right)=\gamma(a, b, c)$ for some $\gamma \in G$. 
Outline of Proof. A real Möbius transformation mapping $B_{X(a, b, c)}=\{\infty, a$, $b, c, i,-i\}$ onto $B_{X\left(a^{\prime}, b^{\prime}, c^{\prime}\right)}=\left\{\infty, a^{\prime}, b^{\prime}, c^{\prime}, i,-i\right\}$ preserves the set $\{i,-i\}$. There exist 8 such Möbius transformations, half of them with real liftings. Namely, (writing their formulae in terms of $a, b$ and $c$ ):

$$
m_{1}(x)=x, \quad m_{2}(x)=\frac{a x+1}{x-a}, \quad m_{3}(x)=\frac{b x+1}{-x+b} \text { and } m_{4}(x)=\frac{c x+1}{x-c} .
$$

Calculating then $m_{j}(\{\infty, a, b, c, i,-i\})$ for each $m_{j}$ and arranging in increasing order the finite real values, we get the points $\left(a^{\prime}, b^{\prime}, c^{\prime}\right) \in T$ we are looking for. They are precisely the images of $(a, b, c)$ under the elements of $G$.

The generating involutions $\alpha$ and $\beta$ act as two reflections with respect to the surfaces $\operatorname{fix}(\alpha)=\{b=(a c+1) /(c-a)\}$ and $\operatorname{fix}(\beta)=\{b=(a c+$ $1) /(a-c)\}$, which intersect along a curve in $T$. It follows that $\Delta=$ $\{(a c+1)(a-c) \leq b \leq(a c+1)(c-a)\}$ is a fundamental set for the action of $G$ in $T$. This proves half of the following theorem.

Theorem 4.3. The mapping

$$
\Psi:(a, b, c) \mapsto X(a, b, c)=\left\{y^{2}=\left(x^{2}+1\right)(x-a)(x-b)(x-c)\right\}
$$

is a bijection between

$$
\Delta=\left\{(a, b, c) \in \mathbb{R}^{3}: a<b<c, \quad \frac{a c+1}{a-c} \leq b \leq \frac{a c+1}{c-a}\right\} \text { and } \mathcal{M}_{(2,2,1)} .
$$

The subsets $D=\left\{\frac{a c+1}{a-c}<b<\frac{a c+1}{c-a}\right\}, S=\left\{\frac{a c+1}{a-c}=b<\frac{a c+1}{c-a}\right\} \cup\left\{\frac{a c+1}{a-c}<\right.$ $\left.b=\frac{a c+1}{c-a}\right\}$ and $C=\{a c+1=b=0\}$ correspond, via $\Psi$, to $\mathcal{M}_{(2,2,1)}\left(C_{2}\right)$, $\mathcal{M}_{(2,2,1)}\left(D_{2}\right)$ and $\mathcal{M}_{(2,2,1)}\left(D_{4}\right)$ respectively. These are the only strata of $\mathcal{M}_{(2,2,1)}$. Furthermore, the explicit formulae of the automorphisms of the curves belonging to each stratum are given.

Proof. The only Möbius transformations with real liftings which may preserve $B_{X(a, b, c)}$ are those appearing in the proof of Proposition 4.2. With the same notations:

- $m_{2}(x)=\frac{a x+1}{x-a}$ preserves $B_{X(a, b, c)}$ if and only if $b=\frac{a c+1}{c-a}$.

- $m_{3}(x)=\frac{b x+1}{-x+b}$ preserves $B_{X(a, b, c)}$ if and only if $a c+1=b=0$.

- $m_{4}(x)=\frac{c x+1}{x-c}$ preserves $B_{X(a, b, c)}$ if and only if $b=\frac{a c+1}{a-c}$.

Let

$$
\begin{aligned}
& f:(x, y) \mapsto\left(\frac{c x+1}{x-c}, \frac{y\left(c^{2}+1\right)^{3 / 2}}{(x-c)^{3}}\right) \\
& g:(x, y) \mapsto\left(\frac{a x+1}{x-a}, \frac{y\left(a^{2}+1\right)^{3 / 2}}{(x-a)^{3}}\right)
\end{aligned} \quad \text { and }
$$


be liftings of $m_{4}$ and $m_{2}$ respectively. Denoting $p=(a, b, c)$ for short, we conclude:

(i) If $p \in D=\left\{\frac{a c+1}{a-c}<b<\frac{a c+1}{c-a}\right\}$ then $\operatorname{Aut} X(p)_{\widehat{\mathbb{C}}}=\left\{\mathrm{id}_{\widehat{\mathbb{C}}}\right\}$ and so $\operatorname{Aut} X(p)=\left\langle h_{X}\right\rangle \simeq C_{2}$.

(ii) If $p \in\left\{\frac{a c+1}{a-c}=b<\frac{a c+1}{c-a}\right\}$ then $\operatorname{Aut} X(p)_{\widehat{\mathbb{C}}}=\left\{\mathrm{id}_{\widehat{\mathbb{C}}}, m_{4}\right\}$ and so $\operatorname{Aut} X(p)$ $=\left\langle h_{X}, f\right\rangle \simeq D_{2}$. If $p \in\left\{\frac{a c+1}{a-c}<b=\frac{a c+1}{c-a}\right\}$ then $\operatorname{Aut} X(p)_{\widehat{\mathbb{C}}}=\left\{\operatorname{id}_{\widehat{\mathbb{C}}}, m_{2}\right\}$ and so $\operatorname{Aut} X(p)=\left\langle h_{X}, g\right\rangle \simeq D_{2}$.

(iii) If $p \in C=\{a c+1=b=0\}$ then $\operatorname{Aut} X(p)_{\widehat{\mathbb{C}}}=\left\{\operatorname{id}_{\widehat{\mathbb{C}}}, m_{2}, m_{3}, m_{4}\right\}$. In this case $\operatorname{Aut} X(p)=\langle f, g\rangle \simeq D_{4}$.

Figure 2 illustrates what $\Delta$ looks like (dashed lines do not belong to $\Delta$ ). The stratum $\mathcal{M}_{(2,2,1)}\left(C_{2}\right)$ corresponds to the interior of $\Delta$ in $T$, which is the domain bounded by the two shadowed surfaces.

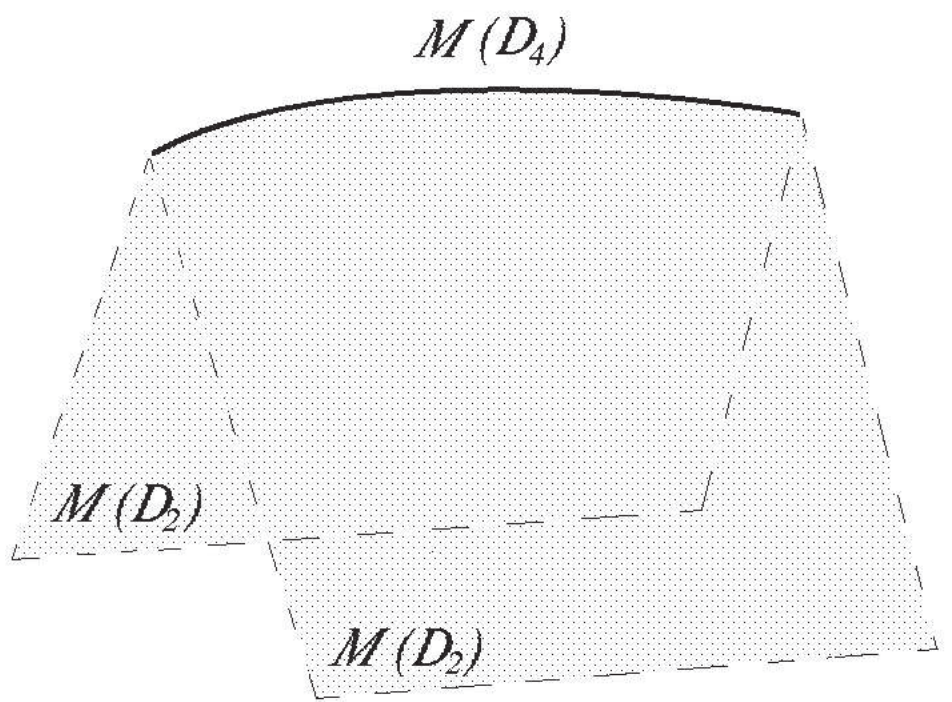

Figure 2. The moduli space of $(2,2,1)$-curves.

\section{Moduli of $(2,1,1)$-curves.}

These curves have two real branching points and four complex non-real ones. We may fix 0 and $\infty$ as the real ones and the imaginary part of two of the others. 
Proposition 5.1. Write $T=\{b>0,(a, b) \neq(c, 1)\} \subset \mathbb{R}^{3}$. The mapping $T \rightarrow \mathcal{M}_{(2,1,1)}$ given by $(a, b, c) \mapsto X(a, b, c)=\left\{y^{2}=x\left((x-a)^{2}+b^{2}\right)((x-\right.$ $\left.\left.c)^{2}+1\right)\right\}$ is surjective.

Its fibres are calculated in the next proposition. Its proof is similar to that of Proposition 4.2 and so we just indicate the formulae of the unique 4 Möbius transformations with real liftings mapping $B_{X(a, b, c)}$ onto $B_{X\left(a^{\prime}, b^{\prime}, c^{\prime}\right)}$. Apart from the identity, they are the following:

$$
m_{2}(x)=\frac{x}{b}, \quad m_{3}(x)=\frac{c^{2}+1}{x}, \text { and } m_{4}(x)=\frac{a^{2}+b^{2}}{b x} .
$$

Proposition 5.2. Let $G$ be the dihedral group of order 4 generated by the involutions

$$
\begin{aligned}
& \alpha:(a, b, c) \mapsto\left(a \cdot \frac{c^{2}+1}{a^{2}+b^{2}}, b \cdot \frac{c^{2}+1}{a^{2}+b^{2}}, c\right) \quad \text { and } \\
& \beta:(a, b, c) \mapsto\left(\frac{c}{b} \cdot \frac{a^{2}+b^{2}}{c^{2}+1}, \frac{1}{b} \cdot \frac{a^{2}+b^{2}}{c^{2}+1}, \frac{a}{b}\right) .
\end{aligned}
$$

Then $X\left(a^{\prime}, b^{\prime}, c^{\prime}\right)$ is isomorphic to $X(a, b, c)$ if and only if $\left(a^{\prime}, b^{\prime}, c^{\prime}\right)=\gamma(a, b, c)$ for some $\gamma \in G$.

A fundamental set for the action of $G$ on $T$ is the set $\Delta$ appearing in the next theorem.

Theorem 5.3. The mapping

$$
\Psi:(a, b, c) \mapsto X(a, b, c)=\left\{y^{2}=x\left((x-a)^{2}+b^{2}\right)\left((x-c)^{2}+1\right)\right\}
$$

is a bijection between

$$
\Delta=\left\{(a, b, c) \in \mathbb{R}^{3}: b>0,(a, b) \neq(c, 1), a \geq b c, a^{2}+b^{2} \geq c^{2}+1\right\}
$$

and $\mathcal{M}_{(2,1,1)}$. The subsets $D=\left\{a>b c, a^{2}+b^{2}>c^{2}+1\right\}$ and $S=\{a=$ $b c\} \cup\left\{a^{2}+b^{2}=c^{2}+1\right\}$ correspond, via $\Psi$, to $\mathcal{M}_{(2,1,1)}\left(C_{2}\right)$ and $\mathcal{M}_{(2,1,1)}\left(D_{2}\right)$ respectively. These are the only strata of $\mathcal{M}_{(2,1,1)}$. Furthermore, the explicit formulae of the automorphisms of the curves belonging to each stratum are given.

Proof. We prove the claims concerning the strata. The only Möbius transformations with real liftings which may preserve $B_{X(a, b, c)}$ are given above. With the same notations:

- $m_{2}(x)=x / b$ preserves $B_{X(a, b, c)}$ if and only if $a=c$ and $b=1$. However, $a, b$ and $c$ cannot take these values.

- $m_{3}(x)=\left(c^{2}+1\right) / x$ preserves $B_{X(a, b, c)}$ if and only if $a^{2}+b^{2}=c^{2}+1$.

- $m_{4}(x)=\left(a^{2}+b^{2}\right) /(b x)$ preserves $B_{X(a, b, c)}$ if and only if $a=b c$. 
Let

$$
\begin{aligned}
& f:(x, y) \mapsto\left(\frac{b\left(c^{2}+1\right)}{x}, \frac{y\left(b\left(c^{2}+1\right)\right)^{3 / 2}}{x^{3}}\right) \quad \text { and } \\
& g:(x, y) \mapsto\left(\frac{c^{2}+1}{x}, \frac{y\left(c^{2}+1\right)^{3 / 2}}{x^{3}}\right)
\end{aligned}
$$

be liftings of $m_{4}$ and $m_{3}$ respectively. Taking into account that no point $p=(a, b, c) \in \Delta$ fulfills simultaneously the equations $a=b c$ and $a^{2}+b^{2}=$ $c^{2}+1$, we get:

(i) If $p \in D=\left\{a>b c, a^{2}+b^{2}>c^{2}+1\right\}$ then Aut $X(p)_{\widehat{\mathbb{C}}}=\left\{\operatorname{id}_{\widehat{\mathbb{C}}}\right\}$, and so Aut $X(p)=\left\langle h_{X}\right\rangle \simeq C_{2}$.

(ii) If $p \in\{a=b c\}$ then $\operatorname{Aut} X(p)_{\widehat{\mathbb{C}}}=\left\{\mathrm{id}_{\widehat{\mathbb{C}}}, m_{4}\right\}$ and so $\operatorname{Aut} X(p)=$ $\left\langle h_{X}, f\right\rangle \simeq D_{2}$. If $p \in\left\{a^{2}+b^{2}=c^{2}+1\right\}$ then $\operatorname{Aut} X(p)_{\widehat{\mathbb{C}}}=\left\{\operatorname{id}_{\widehat{\mathbb{C}}}, m_{3}\right\}$ and so $\operatorname{Aut} X(p)=\left\langle h_{X}, g\right\rangle \simeq D_{2}$.

Figure 3 illustrates what $\Delta$ looks like (dashed lines do not belong to $\Delta$ ). Note that, unlike the preceding cases, the closures in $\Delta$ of the two surfaces corresponding to $\mathcal{M}_{(2,2,1)}\left(D_{2}\right)$ do not intersect.

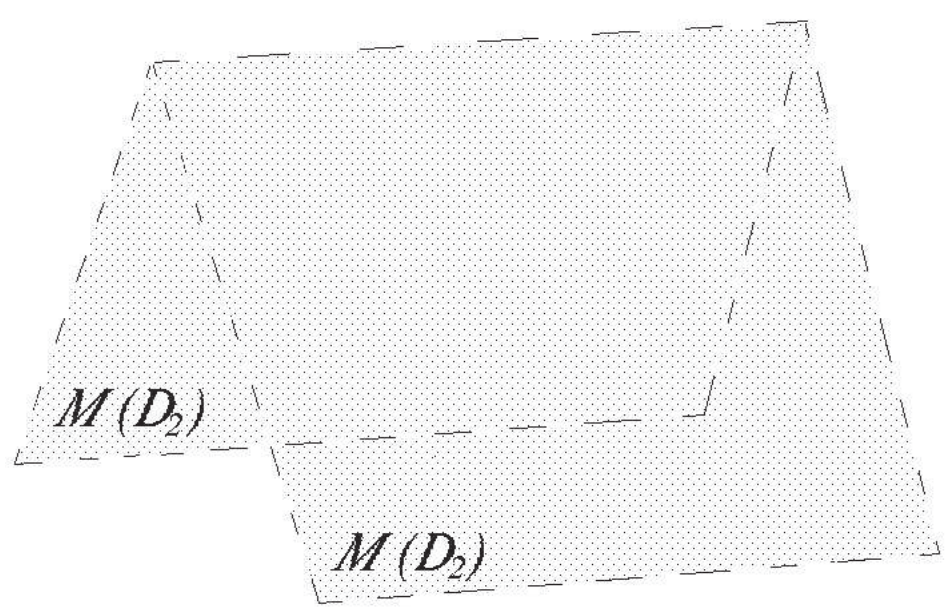

Figure 3. The moduli space of $(2,1,1)$-curves. 


\section{Moduli of $(2,1,0)$ and $(2,0,1)$-curves.}

We may study simultaneously the moduli sets of $(2,1,0)$ and $(2,0,1)$-curves. Indeed, as a consequence of the Topological Classification Lemma, if $P_{X}$ is a monic real polynomial of degree 6 with no real root then $X=\left\{y^{2}=P_{X}\right\}$ is a $(2,1,0)$-curve (and any such curve is like this) while $X^{\prime}=\left\{y^{2}=-P_{X}\right\}$ is a $(2,0,1)$-curve (and any such curve is like this). Therefore, roots of a polynomial as the above serve as parameters to describe the moduli sets of curves of both types. Furthermore, concerning the classification up to isomorphism, an easy computation shows the following.

Lemma 6.1. A mapping $f: X \rightarrow Y$ is an isomorphism between $X=$ $\left\{y^{2}=P_{X}\right\}$ and $Y=\left\{y^{2}=P_{Y}\right\}$ if and only if $f$ is an isomorphism between $X^{\prime}=\left\{y^{2}=-P_{X}\right\}$ and $Y^{\prime}=\left\{y^{2}=-P_{Y}\right\}$. In particular, the automorphism group of $X$ coincides with that of $X^{\prime}$.

Consequently, along this section we only need to deal with $(2,1,0)$-curves, for instance.

Remark 6.2. Curves of these topological types are described by polynomials which are positive on $\mathbb{R}$. In particular $\infty$ is not a branch point and so the $\mathbb{R}$-lifting lemma is superfluous along this section: Every real Möbius transformation mapping the branch point set of $X$ onto that of $Y$ has real liftings.

The description of $\mathcal{M}_{(2,1,0)}$ and $\mathcal{M}_{(2,0,1)}$ by means of the real and imaginary parts of roots of $P_{X}$ is more involved than in the preceding sections. However, the fact that no branch point of $X$ lies in the real axis $\mathbb{R} \cup\{\infty\}$ allows us to consider hyperbolic distances between those with positive imaginary part. The advantage of working with such distances is that they are preserved by real Möbius transformations. Indeed, restrictions to the upper half plane $\mathcal{H}$ of those with positive determinant are the direct isometries of $\mathcal{H}$, whilst the composite of the complex conjugation with those having negative determinant are its inverse ones. Now, the 3 branch points of a $(2,1,0)$-curve lying on $\mathcal{H}$ define a hyperbolic triangle or a hyperbolic segment. Therefore, since here the $\mathbb{R}$-lifting lemma is superfluous, two $(2,1,0)$ curves are isomorphic if and only if their corresponding hyperbolic triangles (or segments) are $\mathcal{H}$-isometric.

It is clear that any hyperbolic triangle or hyperbolic segment is isometric to another with vertices $i$, ai and $b+c i$ for some $a \in(0,1)$ and $b \geq 0$. We can now introduce the following parameters:

$$
\delta_{1}=\rho(i, a i), \quad \delta_{2}=\rho(a i, b+c i), \quad \delta_{3}=\rho(i, b+c i),
$$

where $\rho$ is the hyperbolic distance. Note that $0<\delta_{i} \leq \delta_{j}+\delta_{k}$, for $\{i, j, k\}=\{1,2,3\}$ because they are distances between different points. This way, each isomorphism class of a $(2,1,0)$-curve may be represented by a 
triple $\left(\delta_{1}, \delta_{2}, \delta_{3}\right)$. In order to get unicity in this representation we impose $\delta_{1} \leq \delta_{2} \leq \delta_{3}$. This shows that

$$
\Delta_{\rho}=\left\{0<\delta_{1} \leq \delta_{2} \leq \delta_{3} \leq \delta_{1}+\delta_{2}\right\}
$$

is in bijective correspondence with $\mathcal{M}_{(2,1,0)}$.

We now recover the real and imaginary parts of roots of polynomials defining $(2,1,0)$-curves as the parameters to describe $\mathcal{M}_{(2,1,0)}$. For that we translate the restrictions defining $\Delta_{\rho}$ to restrictions on $a, b$ and $c$. Using the explicit formula for the hyperbolic distance

$$
\rho(z, w)=\ln \left\{\frac{|z-\bar{w}|+|z-w|}{|z-\bar{w}|-|z-w|}\right\}
$$

we get the expressions of $\delta_{1}, \delta_{2}$ and $\delta_{3}$ in terms of $a, b$ and $c$. Then, easy computations give:

$-\delta_{1} \leq \delta_{2}$ if and only if $\left(a^{2}-c\right)(c-1) \leq b^{2}$,

$-\delta_{2} \leq \delta_{3}$ if and only if $b^{2} \leq a-c^{2}$,

- $\delta_{3} \leq \delta_{1}+\delta_{2}$ always, and $\delta_{3}=\delta_{1}+\delta_{2}$ if and only if $b=0$.

Note that if $\delta_{3}=\delta_{1}+\delta_{2}$ then ai lies in the interior of the segment joining $i$ with $c i$; that is, if $b=0$ then $0<c<a<1$, and so in this case condition $\delta_{1} \leq \delta_{2}$ is equivalent to $c \leq a^{2}$.

We can now formulate the main result of this section:

Theorem 6.3. The mapping

$$
\Psi:(a, b, c) \mapsto X(a, b, c)=\left\{y^{2}=\left(x^{2}+1\right)\left(x^{2}+a^{2}\right)\left((x-b)^{2}+c^{2}\right)\right\},
$$

(respectively

$$
\left.\Psi:(a, b, c) \mapsto X^{\prime}(a, b, c)=\left\{y^{2}=-\left(x^{2}+1\right)\left(x^{2}+a^{2}\right)\left((x-b)^{2}+c^{2}\right)\right\}\right),
$$

is a bijection between

$$
\begin{aligned}
\Delta=\{0<a<1, b \geq 0, c>0,(0, a) \neq & (b, c) \neq(0,1), \\
& \left.\left(a^{2}-c\right)(c-1) \leq b^{2} \leq a-c^{2}\right\}
\end{aligned}
$$

and $\mathcal{M}_{(2,1,0)}$ (respectively $\left.\mathcal{M}_{(2,0,1)}\right)$. Furthermore, the subsets of $\Delta$

$$
\begin{aligned}
D= & \left\{b>0,\left(a^{2}-c\right)(c-1)<b^{2}<a-c^{2}\right\}, \\
S= & \left\{b>0,\left(a^{2}-c\right)(c-1)=b^{2}<a-c^{2}\right\} \cup \\
& \left\{b>0,\left(a^{2}-c\right)(c-1)<b^{2}=a-c^{2}\right\} \cup \\
& \left\{b=0, c<a^{2}\right\}, \\
C= & \left\{b=0, c=a^{2}\right\} \quad \text { and } \\
L= & \left\{b>0,\left(a^{2}-c\right)(c-1)=b^{2}=a-c^{2}\right\}
\end{aligned}
$$

correspond, via $\Psi$, respectively to $\mathcal{M}_{(2,1,0)}\left(C_{2}\right), \mathcal{M}_{(2,1,0)}\left(D_{2}\right), \mathcal{M}_{(2,1,0)}\left(D_{4}\right)$ and $\mathcal{M}_{(2,1,0)}\left(D_{6}\right)$ (respectively to $\mathcal{M}_{(2,0,1)}\left(C_{2}\right), \mathcal{M}_{(2,0,1)}\left(D_{2}\right), \mathcal{M}_{(2,0,1)}\left(D_{4}\right)$ and $\left.\mathcal{M}_{(2,0,1)}\left(D_{6}\right)\right)$. These are the only strata of $\mathcal{M}_{(2,1,0)}$ (respectively of 
$\left.\mathcal{M}_{(2,0,1)}\right)$. Furthermore, the explicit formulae of the automorphisms of the curves belonging to each stratum are given.

Proof. In order to calculate the automorphism group of $X(a, b, c)$ we have to find the isometry group of the hyperbolic triangle (or segment) determined by $i$, ai and $b+c i$.

Case 1. If $\delta_{3}=\delta_{1}+\delta_{2}$ then $i$, ai and $c i$ determine a segment with $a i$ in its interior. So any isometry $s$ of this segment fixes $a i$.

1.1: If $s$ also fixes $i$ and $c i$ then $s$ is either the identity or the reflection $x \mapsto-\bar{x}$ in the imaginary axis.

1.2: If $s$ interchanges $i$ and $c i$ then $s$ is the reflection in the $\mathcal{H}$-line orthogonal to the imaginary axis at $a i$, i.e., $x \mapsto a^{2} / \bar{x}$. Note that this case happens only if $\delta_{1}=\delta_{2}$.

Case 2. If $\delta_{3} \neq \delta_{1}+\delta_{2}$ then $i$, ai and $b+c i$ determine a hyperbolic triangle.

2.1: If $\delta_{1}<\delta_{2}<\delta_{3}$ then the triangle is scalene, and so only the identity preserves it.

2.2: If $\delta_{1}=\delta_{2}<\delta_{3}$ then the triangle is isosceles non-equilateral; the only nontrivial isometry that preserves it is the reflection in the angle bisector at ai, i.e., $x \mapsto\left(b \bar{x}+a^{2}(1-c)\right) /((1-c) \bar{x}-b)$.

2.3: If $\delta_{1}<\delta_{2}=\delta_{3}$ then the triangle is also isosceles non-equilateral; the only nontrivial isometry that preserves it is the reflection in the angle bisector at $b+c i$, i.e., $x \mapsto a / \bar{x}$.

2.4: If $\delta_{1}=\delta_{2}=\delta_{3}$ then the triangle is equilateral and so its isometry group is generated by the two reflections described above.

Viewing isometries of $\mathcal{H}$ as real Möbius transformations we obtain the corresponding liftings:

$$
\begin{aligned}
& f_{1}:(x, y) \mapsto(-x, y), \quad f_{2}:(x, y) \mapsto\left(\frac{a^{2}}{x}, \frac{y a^{3}}{x^{3}}\right), \\
& f_{3}:(x, y) \mapsto\left(\frac{a}{x}, \frac{y a^{3 / 2}}{x^{3}}\right) \quad \text { and } \\
& f_{4}:(x, y) \mapsto\left(\frac{b x+a^{2}(1-c)}{(1-c) x-b}, \frac{y \cdot\left[\left(1-a^{2}\right)\left(c-c^{2}\right)\right]^{3 / 2}}{[(1-c) x-b]^{3}}\right) .
\end{aligned}
$$

Translating conditions on $\delta_{1}, \delta_{2}$ and $\delta_{3}$ into conditions of $a, b$ and $c$ we get the following, where $p=(a, b, c)$ for short:

(i) If $p \in\left\{b>0,\left(a^{2}-c\right)(c-1)<b^{2}<a-c^{2}\right\}$ then $\operatorname{Aut} X(p)=\left\langle h_{X}\right\rangle \simeq C_{2}$.

(ii) If $p \in\left\{b=0, c<a^{2}\right\}$ then $\operatorname{Aut} X(p)=\left\langle h_{X}, f_{1}\right\rangle \simeq D_{2}$. If $p \in\{b>$ $\left.0,\left(a^{2}-c\right)(c-1)=b^{2}<a-c^{2}\right\}$ then $\operatorname{Aut} X(p)=\left\langle h_{X}, f_{4}\right\rangle \simeq D_{2}$. If $p \in\left\{b>0,\left(a^{2}-c\right)(c-1)<b^{2}=a-c^{2}\right\}$ then $\operatorname{Aut} X(p)=\left\langle h_{X}, f_{3}\right\rangle \simeq$ $D_{2}$. 
(iii) If $p \in\left\{b=0, a^{2}=c\right\}$ then $\operatorname{Aut} X(p)=\left\langle f_{1}, f_{2}\right\rangle \simeq D_{4}$.

(iv) If $p \in\left\{b>0,\left(a^{2}-c\right)(c-1)=b^{2}=a-c^{2}\right\}$ then Aut $X(p)=\left\langle f_{3}, f_{4}\right\rangle \simeq$ $D_{6}$.

In order to see what $\Delta$ looks like, it is easier to think of its description in terms of $\delta_{1}, \delta_{2}$ and $\delta_{3}$, i.e., to think of $\Delta_{\rho}$. Figure 4 illustrates the relative position of the strata of $\Delta_{\rho}$. There are three boundary surfaces, which correspond to $\mathcal{M}_{(2,1,0)}\left(D_{2}\right)$. Their closures intersect pairwise in three lines; two of them correspond to $\mathcal{M}_{(2,1,0)}\left(D_{4}\right)$ and $\mathcal{M}_{(2,1,0)}\left(D_{6}\right)$, whilst the third (the dashed one) does not belong to $\Delta$. The domain bounded by the three shadowed surfaces corresponds to $\mathcal{M}_{(2,1,0)}\left(C_{2}\right)$.

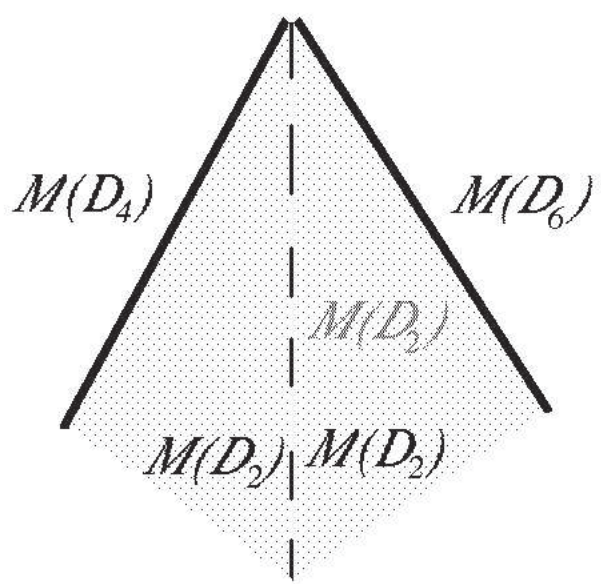

Figure 4. The moduli spaces of $(2,1,0)$ and $(2,0,1)$-curves.

\section{Real analyticity.}

In the preceding sections we gave, for each of the five different topological types of real algebraic curves of genus 2 , a bijection $\Psi_{(2, k, \varepsilon)}: \Delta_{(2, k, \varepsilon)} \rightarrow$ $\mathcal{M}_{(2, k, \varepsilon)}$ between a semialgebraic subset $\Delta_{(2, k, \varepsilon)}$ of $\mathbb{R}^{3}$ and the moduli space $\mathcal{M}_{(2, k, \varepsilon)}$. This latter is known to have a natural semianalytic structure. A natural question arises: Whether the above mapping $\Psi_{(2, k, \varepsilon)}$ is real analytic or not. In this section we answer the question in the affirmative.

Theorem 7.1. Each bijection $\Psi_{(2, k, \varepsilon)}: \Delta_{(2, k, \varepsilon)} \rightarrow \mathcal{M}_{(2, k, \varepsilon)}$ described in the preceding sections is real analytic. 
Let us begin with the case of $(2,3,0)$-curves. With the notations of Section 3 , consider the real analytic manifold $T_{(2,3,0)}=T=\left\{(a, b, c) \in \mathbb{R}^{3}: 0<\right.$ $0<b<c<1\}$. It contains $\Delta_{(2,3,0)}$ and the mapping $\Psi_{(2,3,0)}$ extends to $T$ in the obvious way. Of course, it suffices to show that this extension is real analytic. Let $\mathcal{R}$ be the sheaf of real analytic functions on $T$ and consider the polynomial $f \in \mathcal{R}(T)[X, Y]$ given by

$$
f=Y^{2}-X(X-1)\left(X-u_{1}\right)\left(X-u_{2}\right)\left(X-u_{3}\right),
$$

where each $u_{i} \in \mathcal{R}(T)$ is the $i$ th projection. Consider, with the notations in [7, Section 22], the triple $(\mathcal{C}, \mathcal{U}, \varphi)$ where $\mathcal{U}$ is the trivial open covering of $T$, $\mathcal{C}$ is given by

$$
\mathcal{C}(T)=\frac{\mathcal{R}(T)[X, Y]}{(f)}
$$

and the gluing data $\varphi$ is superfluous here.

Notice that for each $p=(a, b, c) \in T$ the fiber $\mathcal{C}_{p}$ coincides with $\mathcal{C}(T) \otimes_{\mathcal{R}(T)}$ $\mathbb{R}$, where $\mathbb{R}$ is considered as an $\mathcal{R}(T)$-algebra via the evaluation map at $p$. Hence, $\mathcal{C}_{p}$ coincides with $\mathbb{R}[X, Y] /\left(f_{p}\right)$, where $f_{p}=Y^{2}-X(X-1)(X-$ $a)(X-b)(X-c)$. Since $p \in T$, the geometric fiber

$$
\operatorname{Spec} \mathcal{C}_{p}(\mathbb{R})=\left\{(x, y) \in \mathbb{R}^{2}: f_{p}(x, y)=0\right\}
$$

is a real algebraic curve of topological type $(2,3,0)$.

Since $f$ is monic it follows that the triple $(\mathcal{C}, \mathcal{U}, \varphi)$ is an analytic family of real algebraic curves of topological type $(2,3,0)$ as defined in [7]. Therefore a direct application of Theorem 22.2 in [7] gives that the map $\Psi_{(2,3,0)}: T \rightarrow$ $\mathcal{M}_{(2,3,0)}$ is real analytic.

For the rest of topological types the proof is exactly the same, changing $f$ by the appropriate polynomial, because the mapping $\Psi_{(2, k, \varepsilon)}$ extends to an open subset $T$ of $\mathbb{R}^{3}$. For $(2,2,1)$ and $(2,1,1)$-curves, we may take $T$ as in Propositions 4.1 and 5.1, respectively. For $(2,1,0)$ and $(2,0,1)$-curves, it is enough to choose as $T$ a sufficiently small open neighbourhood of the subset $\Delta$ defined in Theorem 6.3 to which $\Psi_{(2, k, \varepsilon)}$ extends.

Remark. The image under $\Psi_{(2, k, \varepsilon)}$ of the interior of $\Delta_{(2, k, \varepsilon)}$ is the set of (isomorphism classes of) $(2, k, \varepsilon)$-curves whose full automorphism group has order 2. By Theorem 5.1 in [6] this is the complement in $\mathcal{M}_{(2, k, \varepsilon)}$ of its boundary and so it is a real analytic manifold of pure dimension 3. Hence by the invariance domain theorem, the restriction of $\Psi_{(2, k, \varepsilon)}$ to the interior of $\Delta_{(2, k, \varepsilon)}$ is a homeomorphism onto its image.

Acknowledgments. This is a part of the author's Ph.D., written at the Complutense University of Madrid under the supervision of Professors J. M. Gamboa and E. Bujalance. I wish to express my gratitude to them for their encouragement and patience. I also wish to thank J. Huisman for calling my attention to the naturality of the semianalytic structure of the real moduli space, and for many helpful explanations concerning it. 


\section{References}

[1] N.L. Alling and N. Greenleaf, Foundations of the Theory of Klein Surfaces, Lecture Notes in Math., 219, Springer-Verlag, 1971, MR 48 \#11488, Zbl 0225.30001.

[2] E. Bujalance, J.A. Bujalance, G. Gromadzki and E. Martínez, The groups of automorphims of non-orientable hyperelliptic Klein surfaces without boundary, Proc. Korea Groups, Lecture Notes in Mathematics, 1398, Springer-Verlag, 1989, 43-51, MR 90m:30060, Zbl 0682.20035.

[3] E. Bujalance and J.M. Gamboa, Automorphism groups of algebraic curves of $\mathbb{R}^{n}$ of genus 2, Arch. Math., 42 (1984), 229-237, MR 86c:20045, Zbl 0539.14022.

[4] F.J. Cirre, On the birational classification of hyperelliptic real algebraic curves in terms of their equations, submitted.

[5] B.H. Gross and J. Harris, Real algebraic curves, Ann. Sci. École Norm. Sup., 14 (1981), 157-182, MR 83a:14028, Zbl 0533.14011.

[6] J. Huisman, Real quotient singularities and nonsingular real algebraic curves in the boundary of the moduli space, Compositio Math., 118 (1999), 43-60, MR 2000i:14033, Zbl 0949.14017.

, Real Teichmüller spaces and moduli of real algebraic curves, Contemp. Math., 253 (2000), 145-177, MR 2001d:32017, Zbl 0986.14037.

[8] F. Klein, Über eine neue art von Riemannschen flächen, Math. Annalen, 10 (1876).

[9] _ Ü̈ber realitätsverhältnisse bei der einem beliebigen geschlechte zugehörigen normalkurve der $\varphi$, Math. Annalen, 42 (1893), 1-29.

[10] A.M. Macbeath and D. Singerman, Spaces of subgroups and Teichmüller space, Proc. London Math. Soc., 31 (1975), 211-256, MR 53 \#882, Zbl 0314.32012.

[11] S.M. Natanzon, Klein surfaces, Uspekhi Mat. Nauk, 45(6) (1990), 47-90 (Russian Math. Surveys. 45(6) (1990), 43-108), MR 92i:14029, Zbl 0734.30037.

[12] M. Seppälä, Teichmüller spaces of Klein surfaces, Ann. Acad. Sci. Fenn. Ser. A I Mathematica Dissertationes, 15 (1978), 1-37, MR 80g:32043, Zbl 0407.32010.

[13] M. Seppälä and R. Silhol, Moduli spaces for real algebraic curves and real abelian varieties, Math. Z., 201 (1989), 151-165, MR 90k:14043, Zbl 0645.14012.

[14] M. Seppälä and T. Sorvali, Geometry of Riemann Surfaces and Teichmüller Spaces, Mathematics Studies, 169, North-Holland, 1992, MR 94b:32034, Zbl 0917.32016.

[15] R. Silhol, Compactifications of moduli spaces in real algebraic geometry, Invent. Math., 107 (1992), 151-202, MR 93i:14018, Zbl 0777.14014.

[16] G. Weichold, Über symmetrische Riemannsche flächen und die periodizitätsmodulen der zugerhörigen abelschen normalintegrale erstes gattung, Dissertation, Leipzig, 1883.

Received April 11, 2001. This work was partially supported by DGICYT PB98-0756.

Dep. Matemáticas Fundamentales

Universidad Nacional de Educación a Distancia

MADRID

SPAIN

E-mail address: jcirre@mat.uned.es 